\title{
Deep Ultraviolet Light Emitting Diodes Based on Short Period Superlattices of AIN/AIGa(In)N
}

Sergey A. Nikishin*, Vladimir V. Kuryatkov, Anilkumar Chandolu, Boris A. Borisov, Gela D. KIPSHIDZE, Iftikhor AHMAD ${ }^{1}$, Mark HoLTZ ${ }^{1}$ and Henryk TEMKIN

Department of Electrical and Computer Engineering and Nano Tech Center, Texas Tech University, Lubbock, Texas, 79409-3102, U.S.A. ${ }^{1}$ Department of Physics and Nano Tech Center, Texas Tech University, Lubbock, Texas 79409-1051, U.S.A.

(Received September 8, 2003; accepted September 26, 2003; published November 5, 2003)

We report a systematic study of the optical properties of superlattices of $A 1 N / \mathrm{Al}_{0.08} \mathrm{Ga}_{0.92}(\mathrm{In}) \mathrm{N}$ with periods in the range of $1.25-2.25 \mathrm{~nm}$. The superlattices were grown on sapphire substrates using gas source molecular beam epitaxy with ammonia. Effective bandgaps between $4.5 \mathrm{eV}(276 \mathrm{~nm})$ and $5.3 \mathrm{eV}(234 \mathrm{~nm})$, as determined by optical reflectivity measurements, were obtained by adjusting the barrier and well thickness. These superlattices can be doped n- and p-type. We demonstrate double heterostructure light emitting diodes operating at wavelengths as short as $262 \pm 2 \mathrm{~nm}$. [DOI: 10.1143/JJAP.42.L1362]

KEYWORDS: LED, GSMBE, ultraviolet, AIN, AIGa(In)N, short period superlattice

There has been significant recent progress towards sub$300 \mathrm{~nm}$ light emitting diodes (LEDs) based on $\mathrm{Al}_{x} \mathrm{Ga}_{1-x} \mathrm{~N}$ alloys. $^{1-9)}$ These alloys can be grown across the full composition range permitting bandgaps ranging from $3.4 \mathrm{eV}(x=0)$ to $6.1 \mathrm{eV}(x=1)$. However, device preparation continues to be limited by the difficulty of preparing $\mathrm{p}$ type layers of large bandgap AlGaN. We have shown that this limitation can be overcome by the use of short period superlattices (SPSLs) consisting of AlN barriers (3-5 monolayers thick) and $\mathrm{Al}_{x} \mathrm{Ga}_{1-x}(\mathrm{In}) \mathrm{N}$ wells (2-3 monolayers thick). ${ }^{1-3,8)}$ The effective energy gaps of $\mathrm{AlN} / \mathrm{AlGaN}^{10)}$ and $\mathrm{AlN} / \mathrm{GaN}^{11,12)}$ SLs are known to depend on the well/barrier thickness ratio, the well composition, and the SL period. Our SPSLs containing as many as 400 well-barrier pairs can be grown reproducibly and with excellent structural and optical properties.. ${ }^{2,3,8,13)}$ When doped with $\mathrm{Mg}$, room temperature hole concentrations as high as $1.1 \times 10^{18} \mathrm{~cm}^{-3}$ were obtained in SPSLs with an average AIN content as high as $65 \% .^{2,3,13)}$ In similar SPSLs doped with $\mathrm{Si}$, electron concentrations of $3 \times 10^{19} \mathrm{~cm}^{-3}$, with the room temperature mobility of $10-20 \mathrm{~cm}^{2} / \mathrm{V} \cdot \mathrm{s}$, could be obtained. ${ }^{2,8)}$ Using nand p-type $\mathrm{AlN} / \mathrm{Al}_{0.08} \mathrm{Ga}_{0.92}(\mathrm{In}) \mathrm{N}$ SPSLs, we have reported UV LEDs with peak emission at 280-290 $\mathrm{nm}^{1-3)}$

In this paper we systematically explore the effects of SPSL parameters on optical properties in the short wavelength limit. Based on the results of these investigations, we demonstrate an LED emitting at $262 \mathrm{~nm}$. All samples were grown by gas source molecular beam epitaxy with ammonia on c-plane sapphire. $\mathrm{Si}$, derived from silane, and $\mathrm{Mg}$ were used for n-type and p-type doping, respectively. Details of epitaxial growth and mesa-LED fabrication have been described elsewhere. ${ }^{1-3,8,13)}$ The effective bandgaps of $\mathrm{AlN} / \mathrm{Al}_{0.08} \mathrm{Ga}_{0.92}$ (In)N SPSLs were obtained from optical reflectance. ${ }^{14)}$ Because of the high growth temperature, the InN content in our wells is quite low. Secondary ion mass spectrometry (SIMS) measurements show approximately $10^{17} \mathrm{~cm}^{-3}$ In atoms in the SL structure. ${ }^{13)}$ The amount added is small enough not to reduce the bandgap or to alter the period of the superlattice but it results in improved luminescence efficiency. ${ }^{1,2)}$ It has been argued recently that even small amounts of In in the lattice may have important effect on the interfacial electric field ${ }^{15)}$ and therefore electrical properties of the SL.

*E-mail address: Sergey.Nikishin@coe.ttu.edu

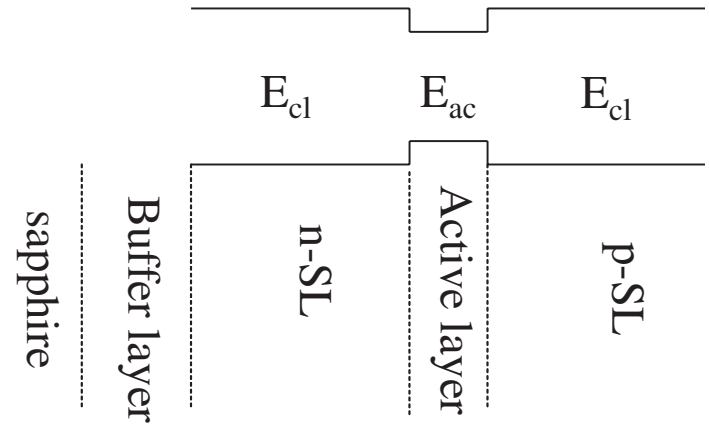

Fig. 1. Schematic cross-section and effective bandgap distribution across DHS LED. Buffer layer is composed of $\mathrm{AlN}$ or $\mathrm{Al}_{0.62} \mathrm{Ga}_{0.38} \mathrm{~N}$. The $E_{\mathrm{cl}}$ and $E_{\mathrm{ac}}$ are the effective bandgaps of n- (p-) type cladding layer and active layer, respectively.

UV devices were designed for emission through the transparent sapphire substrate. The cross-section of basic $\sim 260 \mathrm{~nm}$ double heterostructure (DH) LED and its energy bandgap profile are shown in Fig. 1. Note an each SPSL functions as an artificial crystal having a well-defined effective bandgap. Thus a sandwich of such SPSLs looks similar to a DH composed of bulk semiconductors having different bandgaps. The carrier's injection and optical confinement properties of SPSLs-based DHS are under active investigation. ${ }^{1-3,8,13,16)}$ The LED consists of four main parts: (1) $\mathrm{AlN}$ or $\mathrm{Al}_{0.62} \mathrm{Ga}_{0.38} \mathrm{~N}$ buffer; (2) n-type SPSL cladding layer of $\sim 400 \mathrm{~nm}$; (3) undoped SPSL active region of $\sim 30 \mathrm{~nm}$; (4) p-type SPSL cladding layer of $\sim 210 \mathrm{~nm}$. The buffer layer was incorporated in order to reduce dislocation density in the device SPSL. ${ }^{3)}$ Dislocation density in the top part of the buffer layer was estimated from TEM measurements at $\sim(6-8) \times 10^{9} \mathrm{~cm}^{-2}$. The cladding and active layers were composed of $\mathrm{AlN} / \mathrm{Al}_{0.08} \mathrm{Ga}_{0.92}(\mathrm{In}) \mathrm{N}$ SPSLs having different effective bandgaps. The electron concentration in the n-type SPSL was $\sim 10^{19} \mathrm{~cm}^{-3}$ with the resistivity $\sim 0.04 \Omega \cdot \mathrm{cm}$, measured on a control SPSL grown on sapphire. The p-type Mg-doped SPSL had a total thickness of $\sim 210 \mathrm{~nm}$ and the same composition as the ntype SPSL. The hole concentration of $\sim 10^{18} \mathrm{~cm}^{-3}$ and resistivity of $\sim 4 \Omega \cdot \mathrm{cm}$ were obtained in Hall measurements on SPSL test structures. A 30-nm thick active region was introduced between the $n$ - ans p-type cladding layers to provide carrier confinement. Its bandgap was $\sim 300 \mathrm{meV}$ smaller than that of the cladding layers. The active region 
thickness was chosen to match the electron diffusion lengths measured in p-type SPSLs. ${ }^{16)}$ The bandgap reduction in the active region was accomplished by increasing the well thickness by one monolayer (ML) over that of the $n$ - and $p$ type regions. Note one ML is $\sim 0.25 \mathrm{~nm}$ in AlN or GaN. The introduction of a double heterostructure is an important modification over our previous $280 \mathrm{~nm}$ LED design ${ }^{2,3}$ in which the active region was only $6 \mathrm{~nm}$ thick. In the current design, all the wells are $\mathrm{Al}_{0.08} \mathrm{Ga}_{0.92}$ ( In) N and the barriers are AlN. X-ray diffraction (XRD), cathodoluminescence (CL), and optical reflectance of $\sim 1 \mu \mathrm{m}$ thick test samples of $\mathrm{Al}_{0.08} \mathrm{Ga}_{0.92}$ (In)N were used to measure the well composition and found to be consistent.

We grew two sets of SPSL samples for this study with nominal well thickness of $\sim 0.50$ and $\sim 0.75 \mathrm{~nm}$, i.e. 2 and $3 \mathrm{ML}$. The barrier thickness was varied from $\sim 0.5 \mathrm{~nm}$ to $\sim 1.5 \mathrm{~nm}$, providing a range of corresponding SPSL periods from 1.25 to $2.25 \mathrm{~nm}$. The average composition of the SPSL was determined from the 0th order diffraction using Vegard's Law. The SL period was determined by XRD based on the angular positions of the +1 and -1 SL diffraction peaks. The SPSL $0,-1$, and +1 XRD reflections show slight broadening. Simulations accounting for a $1 \mathrm{ML}$ thick transition region at the barrier-well interfaces adequately describe the broadening seen in the XRD data. The composition of the transition layer depends on the barrier and well growth rates, and the relatively large $\mathrm{Ga}$ and $\mathrm{Al}$ shutter switching times of $\sim 1 \mathrm{~s}$ compared to the $\sim 6 \mathrm{~s}$ well growth time. Detailed XRD simulations, to be published separately, ${ }^{17)}$ indicate that a range of alloy compositions is present in the grown barrier/well interface layers. The analysis suggests that compositions of different $1 \mathrm{ML}$ thick transition layers could change as much as from 30 to $90 \%$ of AIN through the device structure. In order to eliminate a formation of transition layer we plan to decrease the growth rates and reduce shutter switching time.

Figure 2 shows the optical bandgaps, obtained from reflectance measurements, vs. the SPSL period. The effective bandgap is controlled from $\sim 4.5$ to $5.3 \mathrm{eV}$, i.e. from $\sim 276$ to $234 \mathrm{~nm}$, based on the growth parameters. We note some scatter in the data of Fig. 2 and attribute it to several

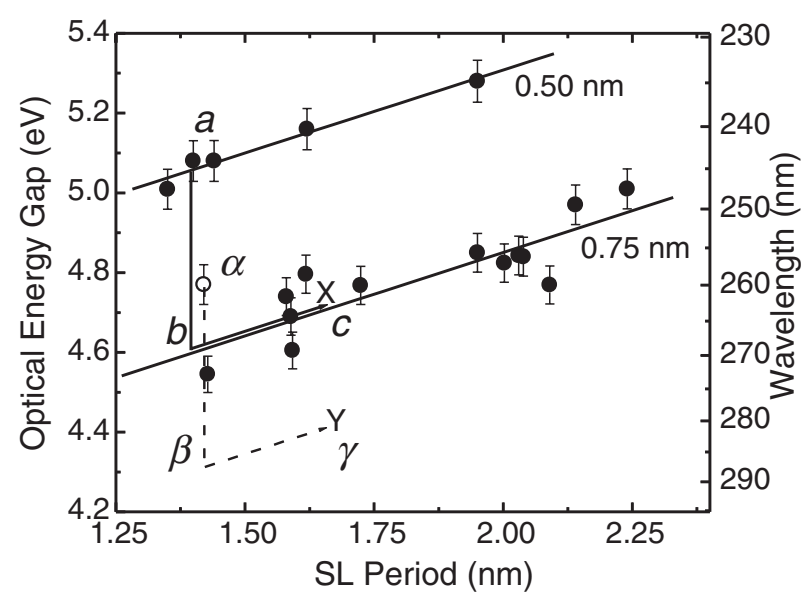

Fig. 2. Effective bandgap of $\mathrm{AlN} / \mathrm{Al}_{0.08} \mathrm{Ga}_{0.92}$ (In)N SPSLs measured by optical reflectance. The upper (lower) data set is for a series of SPSLs with $0.50(0.75) \mathrm{nm}$ well thickness. sources. First, both XRD and optical reflectance measure average properties of the SPSLs and probe regions 20$30 \mathrm{~mm}^{2}$ in area. A second source of scatter in the data is uncertainty in the well and barrier widths due to the growth transition region associated with the shutter switching time, discussed above. A third contributor to variations in the barrier and well thickness, are local composition fluctuations in AlGaN well alloy. ${ }^{18)}$ The optical bandgaps were also found to vary radially by $\pm 2 \mathrm{~nm}$ across the $50 \mathrm{~mm}$ wafer diameter. To minimize the latter effect, we conduct all measurements in the same region of the wafer. In the case of the active regions for our LEDs, the total number of periods is relatively small leading to a much smaller variance. By changing the layer deposition time we were able to deposit SPSLs with well thickness, from XRD, intermediate to the single ML grid. An example is shown in Fig. 2 (open circle near $\alpha$ ), for which the well thickness was found to be $0.68 \mathrm{~nm}$ and period of $1.42 \mathrm{~nm}$. The optical gap for this case was seen to lie between the trends shown for $2 \mathrm{ML}(0.50 \mathrm{~nm})$ and $3 \mathrm{ML}(0.75 \mathrm{~nm})$. We describe later how the intermediate well thickness was used for growing a LED.

The effective bandgap of the SPSL can thus be adjusted either by changing the well or barrier thickness. We observe two trends from the results in Fig. 2, which originate from varying the average AlN composition in the SPSL. First, the $0.50 \mathrm{~nm}$ thinner well series possess energy gaps $400 \pm$ $30 \mathrm{meV}$ higher than the $0.75 \mathrm{~nm}$ well set for the same period. For example, points $a$ and $b$ in Fig. 2 illustrate the effect of increasing the well thickness by one ML and decreasing the barrier thickness by the same amount. The second trend is observed from systematically varying the barrier thickness while maintaining constant well thickness. A linear fit provides a shift $\sim 100 \pm 20 \mathrm{meV} / \mathrm{ML}(\sim 7 \mathrm{~nm} / \mathrm{ML})$ for each well thickness series. We illustrate this in Fig. 2 through points $b$ and $c$. We utilize here a simple linear approximation for guiding our device design. A more complete data set, with additional well thickness, is required to develop a better picture of the dependence of energy gap on SPSL parameters. It is clear from our results that control of the SPSL well and barrier thickness provides "coarse" and "fine" adjustment of the effective bandgap. Changing the well thickness with fixed period provides the coarse control of $400 \pm 30 \mathrm{meV}$. Keeping the well thickness constant and growing with different barrier widths provides the fine control of $100 \pm 20 \mathrm{meV} / \mathrm{ML}$. The two approaches to changing the energy gap may also be used in combination. For example, increasing the well by $1 \mathrm{ML}$ and leaving the barrier thickness constant produces an intermediate change in bandgap energy of $270 \pm 20 \mathrm{meV}$, such as points $a$ and $c$ in Fig. 2. We design our LED cladding and active layers based on these results. The growth procedure of adding one ML to the well is straightforward, and avoids reducing the barrier to two MLs in thickness.

Mesa-etched LEDs were fabricated as previously described. ${ }^{8)}$ The current-voltage (I-V) characteristic of a $160 \mu \mathrm{m}$ mesa LED is plotted in Fig. 3. LED light emission is observed with forward dc current above $2 \mathrm{~mA}$. The device turns on at $\sim 6.0 \mathrm{~V}$ and has differential series resistance $R_{\mathrm{m}}$ of $110-120 \Omega$ under forward bias from $8 \mathrm{~V}$ to $12 \mathrm{~V}$. The $R_{\mathrm{m}}$ of mesa diodes is the sum of the contact $\left(R_{\mathrm{c}}\right)$, spreading $\left(R_{\mathrm{S}}\right)$, and vertical $\left(R_{\mathrm{v}}\right)$ resistances. The resistance of the etched 


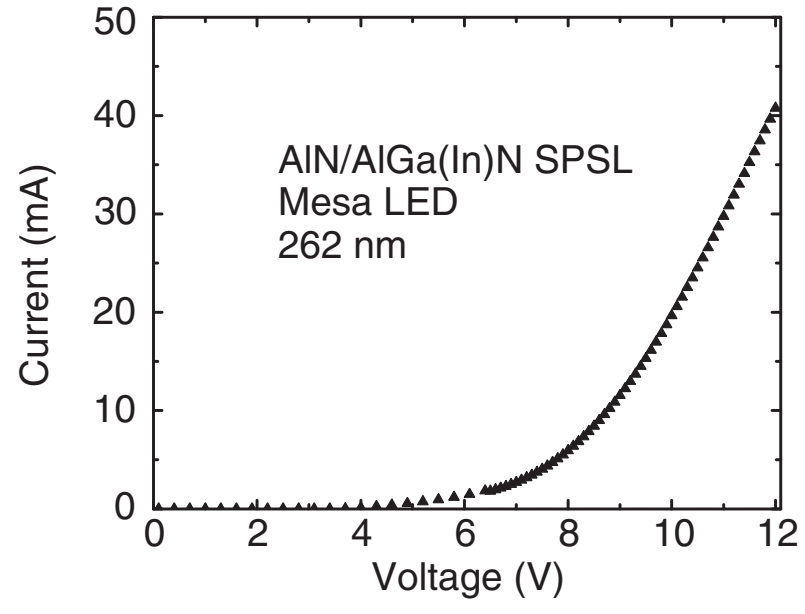

Fig. 3. Room-temperature $I-V$ of a $160 \mu \mathrm{m}$ mesa LED.

part of the mesa, corresponding to transport across the SPSL layers is $R_{\mathrm{V}} \approx \rho_{\perp}(h / A)$, where $\rho_{\perp}$ is the perpendicular resistivity of the SPSL, $h$ is the height of the mesa ( $\sim 300 \mathrm{~nm}$, with the thickness of p-SL $\sim 200 \mathrm{~nm})$, and $A$ is the contact area. The contact resistance of a $160-\mu \mathrm{m}$ diameter diode, obtained from specific contact resistance, is $R_{\mathrm{c}} \sim 90 \Omega$. The estimated spreading resistance of this LED is $R_{\mathrm{S}} \sim 20 \Omega$ and comes from n-type buffer layer. Finally we obtain $R_{\mathrm{V}} \sim 5 \Omega$ resulting in $\rho_{\perp} \sim 50 \Omega \cdot \mathrm{cm}$ for a p-type SPSL. Comparing this to the in-plane conductivity $\left(\rho_{\square}\right)$ of the p-type SPSL obtained from Hall measurements, $\rho_{\square} \sim 4 \Omega \cdot \mathrm{cm}$, we obtain the conductivity anisotropy $\rho_{\perp} / \rho_{\square} \sim 12$. These simple considerations indicate relatively low $\rho_{\perp}$, considering the high AlN fraction in our SPSLs, and underscore the importance of reducing the contact resistance to p-type materials. It is also important to lower $R_{\mathrm{s}}$ by optimization of the buffer layer thickness and its resistivity.

Based on the results of Fig. 2, we produced two LEDs operating at different wavelength. The first uses cladding layers described by point $a$ in Fig. 2. In the active region the well width is increased by $1 \mathrm{ML}$ and the barrier thickness is unchanged, corresponding to point $c$ in Fig. 2. The EL spectrum of this LED is shown in Fig. 4. For the $4 \mathrm{~mA} \mathrm{dc}$

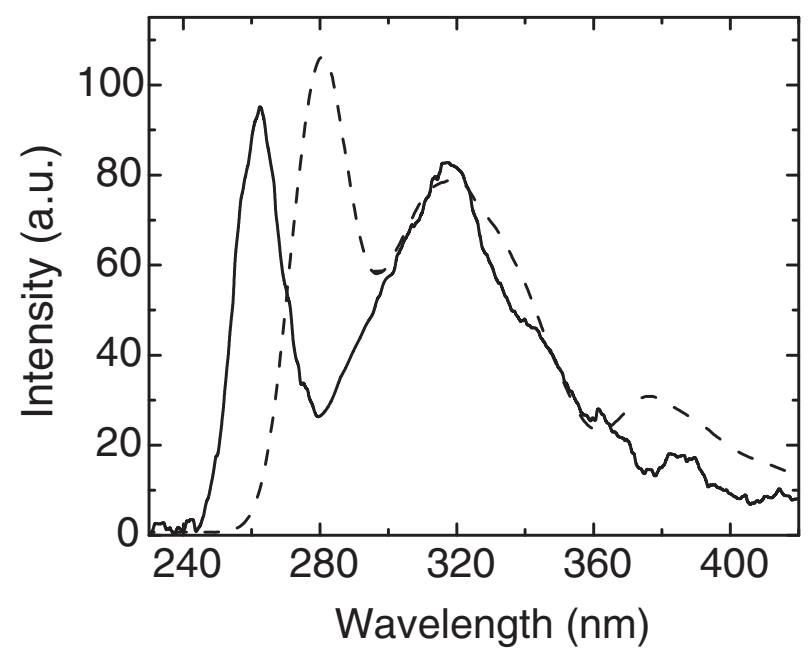

Fig. 4. EL spectra of $262 \mathrm{~nm}$ and $280 \mathrm{~nm}$ LEDs operating at low dc current of $\sim 4 \mathrm{~mA}$. drive current shown, two peaks are observed at wavelengths of 262 and $320 \mathrm{~nm}$. We focus here on the short wavelength peak at $262 \mathrm{~nm}$ and will return to the $320 \mathrm{~nm}$ feature later. We include the peak at $262 \mathrm{~nm}$ (labeled "X") in Fig. 2. Agreement is excellent between the measured emission wavelength and what was expected based on the $1 \mathrm{ML}$ $(a \rightarrow c)$ increase in well thickness. A second LED uses cladding layers with properties illustrated by $\alpha$ in Fig. 2. The average well thickness of the $n$ - and p-type cladding materials, as discussed earlier, is intermediate to 2 and 3 ML. For the active layer we increase the well thickness by $1 \mathrm{ML}$ and leave the barrier thickness intact. This is illustrated by points $\alpha$ and $\gamma$ in Fig. 2, assuming here that the energy differences $\alpha \rightarrow \beta \rightarrow \gamma$ in this analysis are the same as what we used for analogous points $a, b$, and $c$ with integer well thickness. The EL emission spectrum for this LED is also shown in Fig. 4. At the same forward dc current of $4 \mathrm{~mA}$, two peaks at $282 \mathrm{~nm}$ and $320 \mathrm{~nm}$ are observed. The $282 \mathrm{~nm}$ peak is included in Fig. 2 as symbol Y. We see from this that the design approach accurately predicts the short wavelength LED emission energy.

Now we turn our attention to the broad $320 \mathrm{~nm}$ peak seen in both EL spectra in Fig. 4. At low forward current its intensity is almost equal to that of the bandedge emission. However, the bandedge emission grows faster with current, exceeding the intensity of the $320 \mathrm{~nm}$ peak by factors of 2 and 3 at dc currents of $10 \mathrm{~mA}^{9)}$ and $12 \mathrm{~mA}^{2)}$ respectively. A similar feature has been observed in $285 \mathrm{~nm} \mathrm{LEDs}{ }^{19)}$ and $292 \mathrm{~nm} \mathrm{LEDs}^{9)}$ based on random alloy layers and attributed to a free-carrier to deep acceptor transition in Mg-doped p$\mathrm{Al}_{0.4} \mathrm{Ga}_{0.6} \mathrm{~N}$. Such a transition could arise in our SPSL-based LED because of the formation of $1 \mathrm{ML}$ thick barrier/well interfaces in which the AlN concentration may range from $30 \%$ to $90 \%$ of AlN, as mentioned above. Even though the interface regions are only $1 \mathrm{ML}$ thick, the total volume fraction of these regions throughout the SPSL can be considerable since the period is so small. We note however that the position and width of the $320 \mathrm{~nm}$ peak in Fig. 4 do not scale with the effective bandgap of SPSL, making it difficult to assign our feature to a free-carrier to deep acceptor transition. Our CL studies, reported elsewhere, ${ }^{2)}$ show that the intensity of the long wavelength peak scales with Si doping in the SPSLs. Similar sub-bandgap features are observed in $290 \mathrm{~nm}$ LEDs grown on silicon substrates. ${ }^{1)}$ Clearly, additional experiments are required in order to better identify the origin of the $320 \mathrm{~nm}$ peak present in UV LEDs.

To summarize, we use the SPSL approach to engineer bandgaps as large as $5.3 \mathrm{eV}$ and demonstrate DHS LEDs with emission wavelength as short as $262 \mathrm{~nm}$. We systematically vary the properties of $\mathrm{AlN} / \mathrm{Al}_{x} \mathrm{Ga}_{1-x}(\mathrm{In}) \mathrm{N}$ SPSLs to show that absorption edge and emission wavelength are controllable by varying the well and barrier properties. Optical bandgaps could be adjusted reproducibly between $\sim 4.5$ and $5.3 \mathrm{eV}$ by monolayer variations in the well and barrier thickness. Thus the DHS with bandgap offset of $\sim 0.8 \mathrm{eV}$ can be obtained. Emission is observed from the active region in excellent agreement with the design optical bandgap. With cladding layers composed of $0.50 \mathrm{~nm}$ wells and having $\sim 2 \mathrm{~nm}$ period, SPSL-based "homojunction" LEDs with deep UV emission near $240 \mathrm{~nm}$ appear feasible. 
Here "homojunction" means that the effective bandgaps of all SPSLs are the same.

The authors acknowledge support from DARPA-SUVOS (Dr. J. Carrano), NSF (ECS-0070240, ECS-9871290, ECS0323640, and ECS-0304224), U. S. Army SBCCOM, NATO Science for Peace (974505), and the J. F. Maddox Foundation. We also thank Dr. S. N. G. Chu for TEM measurements.

1) G. Kipshidze, V. Kuryatkov, B. Borisov, M. Holtz, S. Nikishin and H. Temkin: Appl. Phys. Lett. 80 (2002) 3682.

2) G. Kipshidze, V. Kuryatkov, K. Zhu, B. Borisov, M. Holtz, S. Nikishin and H. Temkin: J. Appl. Phys. 93 (2003) 1363.

3) G. Kipshidze, V. Kuryatkov, B. Borisov, S. Nikishin, M. Holtz, S. N. G. Chu and H. Temkin: Phys. Status Solidi A 192 (2002) 286.

4) V. Adivarahan, J. Zhang, A. Chitnis, W. Shuai, J. Sun, R. Pachipulusu, M. Shatalov and M. A. Khan: Jpn. J. Appl. Phys. 41 (2002) L435.

5) A. Yasan, R. McClintock, K. Mayers, S. R. Darvish, P. Kung and M. Razeghi: Appl. Phys. Lett. 81 (2002) 801.

6) A. Chitnis, R. Pachipulusu, V. Mandavilli, M. Shatalov, E. Kuokstis, J. P. Zhang, V. Adivarahan, S. Wu, G. Simin and M. A. Khan: Appl. Phys. Lett. 81 (2002) 2938.

7) V. Adivarahan, S. Wu, A. Chitnis, R. Pachipulusu, V. Mandavilli, M. Shatalov, J. P. Zhang, M. A. Khan, G. Tamulaitis, A. Sereika, I Yilmaz, M. S. Shur and R. Gaska: Appl. Phys. Lett. 81 (2002) 3666

8) K. Zhu, V. Kuryatkov, B. Borisov, G. Kipshidze, S. A. Nikishin, H.
Temkin and M. Holtz: Appl. Phys. Lett. 81 (2002) 4688

9) A. Hanlon, P. M. Pattison, J. F. Kaeding, R. Sharma, P. Fini and S. Nakamura: Jpn. J. Appl. Phys. 42 (2003) L628.

10) H. Hirayama, Y. Enomoto, A. Konoshita, A. Hirata and Y. Aoyagi: Appl. Phys. Lett. 80 (2002) 37.

11) Z. Sitar, M. J. Paisley, B. Yan, J. Ruan, W. J. Choyke and R. F. Davis: J. Vac. Sci. \& Technol. B 8 (1990) 316.

12) M. A. Khan, J. N. Kuznia, D. T. Olson, T. George and W. T. Pike Appl. Phys. Lett. 63 (1993) 3470.

13) V. Kuryatkov, K. Zhu, B. Borisov, A. Chandolu, Iu. Gherasoiu, G. Kipshidze, S. N. G. Chu, M. Holtz, Yu. Kudryavtsev, R. Asomoza, S. Nikishin and H. Temkin: Appl. Phys. Lett. 83 (2003) 1319.

14) M. Holtz, T. Prokofyeva, M. Seon, K. Copeland, J. Vanbuskirk, S. Williams, S. A. Nikishin, V. Tretyakov and H. Temkin: J. Appl. Phys. 89 (2001) 7977.

15) I. Lo, J. K. Tsai, L.-W. Tu, K. Y. Hsieh, M. H. Tsai, C. S. Liu, J. H. Huang, S. Elhamri, W. C. Mitchel and J. K. Sheu: Appl. Phys. Lett. 80 (2002) 2684

16) S. A. Nikishin, V. V. Kuryatkov, B. A. Borisov, G. D. Kipshidze, A Chandolu, M. Holtz, L. Chernyak and H. Temkin: Tech. Dig. 5th Int. Conf. Nitride Semiconductors, Nara, 2003, p. 160.

17) A. Chandolu, S. Nikishin and H. Temkin: unpublished.

18) F. Yun, M. A. Reshchikov, L. He, T. King and H. Morkoç: J. Appl. Phys. 92 (2002) 4837.

19) M. Shatalov, A. Chitnis, V. Mandavilli, R. Pachipulusu, J. P. Zhang, V. Adivarahan, S. Wu, G. Simin, M. A. Khan, G. Tamulaitis, A. Sereika, I. Yilmaz, M. S. Shur and R. Gaska: Appl. Phys. Lett. 82 (2003) 167. 